\title{
Improving Data Quality in eBird- the Expert Reviewer Network
}

Steve Kelling $\ddagger$

‡ Cornell Lab of Ornithology, Ithaca, United States of America

Corresponding author: Steve Kelling (stk2@cornell.edu)

Received: 31 Mar 2018| Published: 17 May 2018

Citation: Kelling S (2018) Improving Data Quality in eBird- the Expert Reviewer Network. Biodiversity Information Science and Standards 2: e25394. https://doi.org/10.3897/biss.2.25394

\begin{abstract}
eBird is a global citizen science project that gathers observations of birds. The project has been making a considerable contribution to the collection and sharing of bird observations, even in the data-poorest countries, and is accelerating the accumulation of bird records globally. On 22 March 2018 eBird surpassed $1 / 2$ billion bird observations.

A primary component of ensuring the best quality data is the network of more than 1300 volunteer reviewers who scour incoming data for accuracy. Reviewers provide active feedback to participants on everything from bird identification to best practices for data collection. Since eBird's inception in 2002, almost 23 million observations have been reviewed, requiring more than 190,000 hours of effort by reviewers. In this presentation we review how eBird recruits expert reviewers, describe their responsibilities, and offer some insight in new developments to improve the reviewing process.
\end{abstract}

How are reviewers recruited. There are three primary methods that used to identify new reviewers. First, if we don't have any active participants in a region (e.g., Kamchatka Russia) eBird staff search birding listserves to find an individual who is reporting a lot of high-quality observations from the area. We then contact those individuals and offer them the opportunity to review records for the region. This option has the lowest likelihood of success. Second, if an individual is submitting a lot of records to eBird from a region that needs a reviewer we contact them and request their participation. Third, in much of the world eBird has partner groups. These partner organizations (e.g., Taiwan, Spain, India, 
Portugal, Australia, and all of the Western Hemisphere) recruit their own reviewers. The third method is the most effective way to gain expert participation.

What does a reviewer do? eBird reviewers work to improve eBird data in three primary areas. First, they develop and manage the eBird checklist filters for a region. These filters generate a checklist of birds for a particular time and location, and determine what records get flagged for further review. Second, if an eBird participant tries to report a species that is not on the checklist, or if the number of individuals of a species exceeds the filter limit, then these records get flagged for review. Reviewers contact the observer and request further documentation. Currently, $57 \%$ of all records that are evaluated by reviewers are validated. Finally, eBird reviewers validate whether the participant is eBirding correctly. That is, are they correctly filling out the information on when, where, and how they went birding. It has been our experience that different types of reviewers are required to effectively review eBird submissions: those who are good at reviewing bird records and those who are good at educating observers on how to participate.

What are future plans? eBird will move towards more effective reviewer teams, where the volume of observations can be split amongst a number of individuals with different strengths, allowing identification experts to focus on observation-level ID issues; and strong communicators to focus on working with contributors on checklist-level best practices. Currently, a single eBird review platform handles a broad array of different reviewing functions. It is our intent to split some of these functions into multiple platforms. For example, right now all review happens at the database level of the 'observation': a record of a taxon at a date and location. Plans are underway to develop tools that will allow reviewers to work at the entire checklist level (i.e., to more easily review the accuracy of how all the observations during a checklist event were submitted), which will enable much more effective review of checklist-level data quality concerns.

\section{Keywords}

eBird, data quality, citizen science, data review

\section{Presenting author}

Steve Kelling

\section{Acknowledgements}

We thank the eBird volunteer expert reviewers for their contributions, and the National Science Foundation (ABI sustaining: DBI-1356308). 